
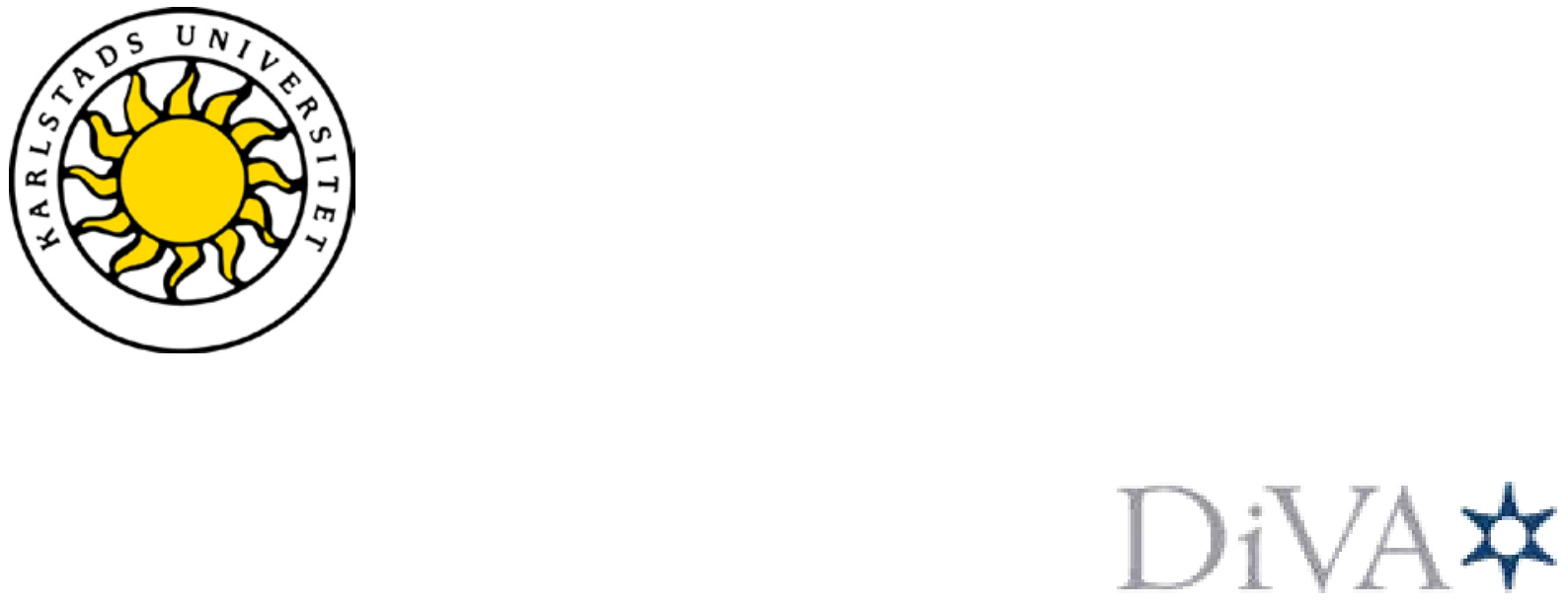

http:/ / kau.diva-portal.org

This is a digital duplicate of the Publisher's version of a paper published in Applied Physics Letters.

This paper has been peer-reviewed and includes the final publisher proof-corrections and journal pagination.

Citation for the published paper:

H. Jackman, P. Krakhmalev and K. Svensson

"Measurements of the critical strain for rippling in carbon nanotubes" Applied Physics letters, 2011, Vol. 98, Issue: 18

http://dx.doi.org/10.1063/1.3587613

Publish with permission from: American Institute of Physics 


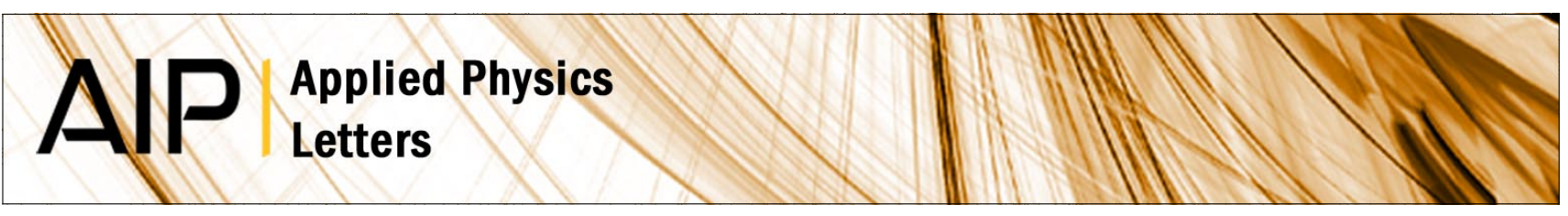

Measurements of the critical strain for rippling in carbon nanotubes

H. Jackman, P. Krakhmalev, and K. Svensson

Citation: Appl. Phys. Lett. 98, 183104 (2011); doi: 10.1063/1.3587613

View online: http://dx.doi.org/10.1063/1.3587613

View Table of Contents: http://apl.aip.org/resource/1/APPLAB/v98/i18

Published by the American Institute of Physics.

\section{Related Articles}

Slippage toughness measurement of soft interface between stiff thin films and elastomeric substrate Rev. Sci. Instrum. 82, 104704 (2011)

On the strain in n-type GaN

Appl. Phys. Lett. 99, 141916 (2011)

Plastic flow in shock-loaded silver at strain rates from $104 \mathrm{~s} 1$ to $107 \mathrm{~s} 1$ and temperatures from $296 \mathrm{~K}$ to $1233 \mathrm{~K}$ J. Appl. Phys. 110, 073502 (2011)

Compliant substrate versus plastic relaxation effects in Ge nanoheteroepitaxy on free-standing $\mathrm{Si}(001)$ nanopillars

Appl. Phys. Lett. 99, 141901 (2011)

Buckling of poly(methyl methacrylate) in stimulus-responsive shape recovery

Appl. Phys. Lett. 99, 131911 (2011)

\section{Additional information on Appl. Phys. Lett.}

Journal Homepage: http://apl.aip.org/

Journal Information: http://apl.aip.org/about/about_the_journal

Top downloads: http://apl.aip.org/features/most_downloaded

Information for Authors: http://apl.aip.org/authors

\section{ADVERTISEMENT}

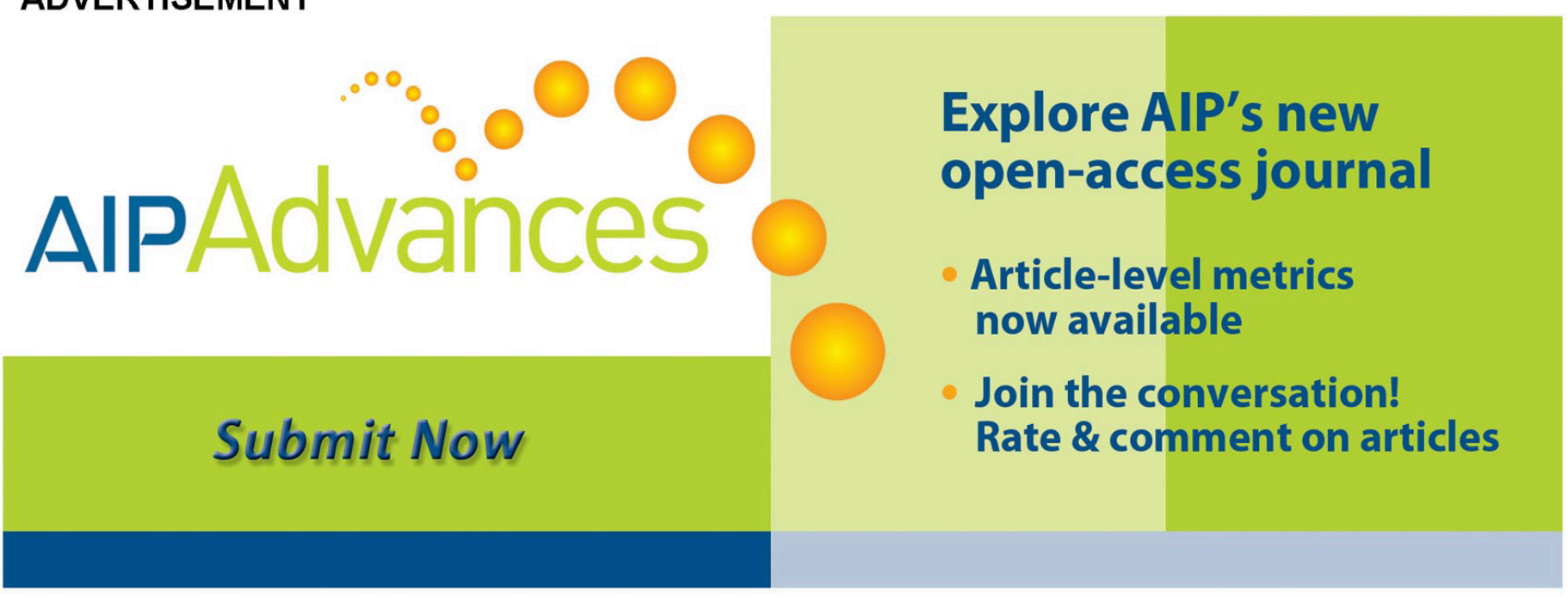




\title{
Measurements of the critical strain for rippling in carbon nanotubes
}

\author{
H. Jackman, ${ }^{1, a)}$ P. Krakhmalev, ${ }^{2}$ and K. Svensson ${ }^{1}$ \\ ${ }^{1}$ Department of Physics and Electrical Engineering, Karlstad University, SE-651 88 Karlstad, Sweden \\ ${ }^{2}$ Department of Mechanical and Materials Engineering, Karlstad University, SE-651 88 Karlstad, Sweden
}

(Received 25 March 2011; accepted 13 April 2011; published online 4 May 2011)

\begin{abstract}
We report measurements of the bending stiffness in free standing carbon nanotubes, using atomic force microscopy inside a scanning electron microscope. Two regimes with different bending stiffness were observed, indicative of a rippling deformation at high curvatures. The observed critical strains for rippling were in the order of a few percent and comparable to previous modeling predictions. We have also found indications that the presence of defects can give a higher critical strain value and a concomitant reduction in Young's modulus. (C) 2011 American Institute of Physics. [doi:10.1063/1.3587613]
\end{abstract}

Carbon nanotubes (CNTs) are promising building blocks in future nanoelectromechanical systems (NEMS) due to their low mass and high Young's modulus, E, thereby enabling high switching frequencies. ${ }^{1,2}$ A detailed understanding of the accommodation of strain in CNTs will be important for such applications. As the nanotubes consist of concentric cylinders and are hollow in the center, they behave differently from solid cylinders. During bending, the nanotubes can deform in two different modes, one is a subtle rippling of the walls ${ }^{3-8}$ and another is a local collapse (buckling) of the walls. ${ }^{9}$ The rippling is the first deformation to appear as a CNT is subjected to bending, while the buckling appears at higher bending curvatures. ${ }^{10}$ The initial bending stiffness will be directly related to Young's modulus, while the bending stiffness in the rippling mode has been predicted to depend on the number of walls. ${ }^{7}$ The rippling mode will emerge at a critical bending strain, $\varepsilon_{c r}$, which has been predicted to depend on the radius of the $\mathrm{CNT}^{5,7}$ Both deformation modes will result in a lower bending stiffness and estimations of $E$ should therefore be done at low bending strains to avoid the influence of the rippling and buckling modes. It has been suggested that the rippling mode could be responsible for the apparent diameter dependence in the reported values of Young's modulus. 6,8 A reduction in the bending stiffness will lower the switching frequency in NEMS applications and a detailed understanding of the deformation modes is therefore valuable for NEMS design.

While the buckling mode is readily observable ${ }^{9}$ in transmission electron microscopy (TEM), the small atomic rearrangements near the onset of the rippling mode would be hard to detect in direct imaging. Far into the rippling mode there are observations though of wavelike deformations. ${ }^{8}$ One way to detect the onset of rippling is to study the force response of the nanotubes under bending, as there should be a distinct reduction in the bending stiffness when the tubes enter the rippling mode. ${ }^{3-7}$ A nonlinear response in CNTs has been observed for tubes deposited on substrates ${ }^{11}$ and it was suggested to be the buckling regime (but might have been due to rippling). Thus far there are no direct experimental observations of $\varepsilon_{c r}$ in free standing tubes.

Here we present direct force measurements on individual, free standing, CNTs inside a scanning electron micro-

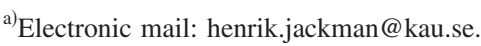

scope (SEM). Distinct changes in the spring constants, indicating a rippling onset, were observed. For strains above $\varepsilon_{c r}$ we have found that the force response continued to be linear, albeit with a lower spring constant. The observed magnitude of $\varepsilon_{c r}$, and the reduction in the bending stiffness, are comparable to previous theoretical modeling.

Measurements were performed using a custom made atomic force microscopy (AFM) instrument with piezoresistive force sensors, ${ }^{12}$ controlled by software and electronics from Nanofactory Instruments. ${ }^{13}$ The motion of the tubescanner in the AFM was calibrated by using the SEM, and the force sensor was calibrated by pushing precalibrated AFM cantilevers against it. ${ }^{14}$ The AFM instrument was mounted inside a LEO 1530 FEG-SEM, operated at an acceleration voltage of $12 \mathrm{kV}$ and a chamber pressure of about $5 \times 10^{-7}$ mbar. Two types of commercial CNTs were investigated in this study, NC2100 and NC2101, both produced by Nanocyl (Ref. 15) via catalytic chemical vapor deposition (CCVD). Both types are marketed as double-walled tubes, and the $\mathrm{NC} 2101$ are functionalized (with-COOH) to reduce bundling. As received samples were dispersed in ethanol and then sonicated for $15 \mathrm{~min}$, in order to separate the tubes without causing too much damage in the process (this was checked with TEM). Droplets of the solution were placed on a glass substrate and allowed to dry. Individual soot particles were picked up (under an optical microscope) using mechanically cut silver tips coated with conducting epoxy glue. The tip was attached to a tube-scanner that enables both coarse and fine motion in three dimensions. ${ }^{16}$ Forcedisplacement, $F$ - $\delta$, curves were obtained by pushing individual CNTs against the AFM cantilever in a cantilever-tocantilever fashion, see Fig. 1.

The resulting $F$ - $\delta$ curves show an initial linear relation with spring constant $k_{i}$ for small displacements (see Fig. 2). At a critical displacement, $\delta_{c r}$, the spring constant abruptly decreases, due to the emergence of the rippling mode. The $F$ - $\delta$ relation still appears to be linear though with a spring constant, $k_{r}$. Upon retraction the behavior was reversible without hysteresis, apart from the snap-out. In Fig. 2 two typical $F-\delta$ curves are shown together with linear fits indicated by dashed lines. Zero displacement of the CNT was taken to be when the force returns to zero after the snap-in, and the displacement for rippling onset was taken to be where the two linear fits intersect. The critical displacement, 

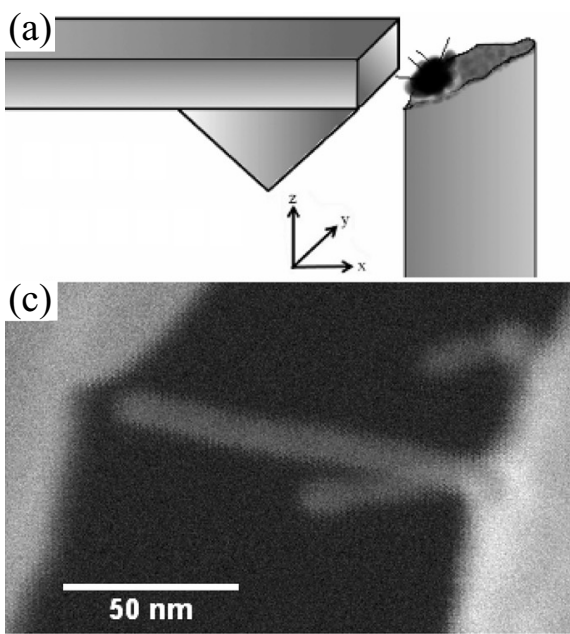

(b)
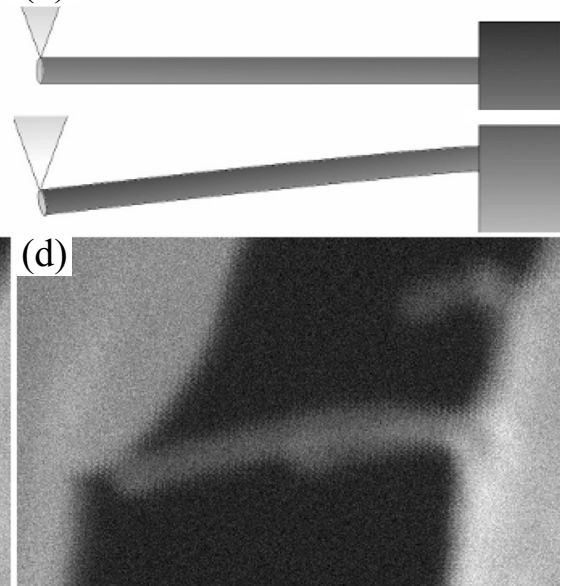

FIG. 1. (a) Experimental set-up with the CNTs glued to a silver tip to the right and the AFM cantilever to the left. (b) Schematic sketch of the setup and (c) an SEM image of a CNT before and (d) during bending. $\delta_{c r}$, was defined as the difference between these two points, as shown in Fig. 2. We have not been able to detect any nonlinearity in the rippling mode, while modeling have found a nonlinear response with a power in the range of $0.42-0.66 .^{5}$

Assuming the response to be linear and comparing the two spring constants, the ratio $k_{r} / k_{i}$ showed no dependence on $r$ and the mean value was found to be 0.56 with a standard deviation of 0.11 . Theoretical modeling have found a variation in values for the ratio, $k_{r} / k_{i}$. In the work of Arroyo and Arias ${ }^{5}$ and Liu et al., ${ }^{6}$ a ratio of about 0.2 can be seen in their figures. On the other hand, Nikiforov et al. ${ }^{7}$ have found a dependence on the number of walls, where $k_{r} / k_{i}$ increases from 0.15 for a double-walled CNT and converges to 0.65

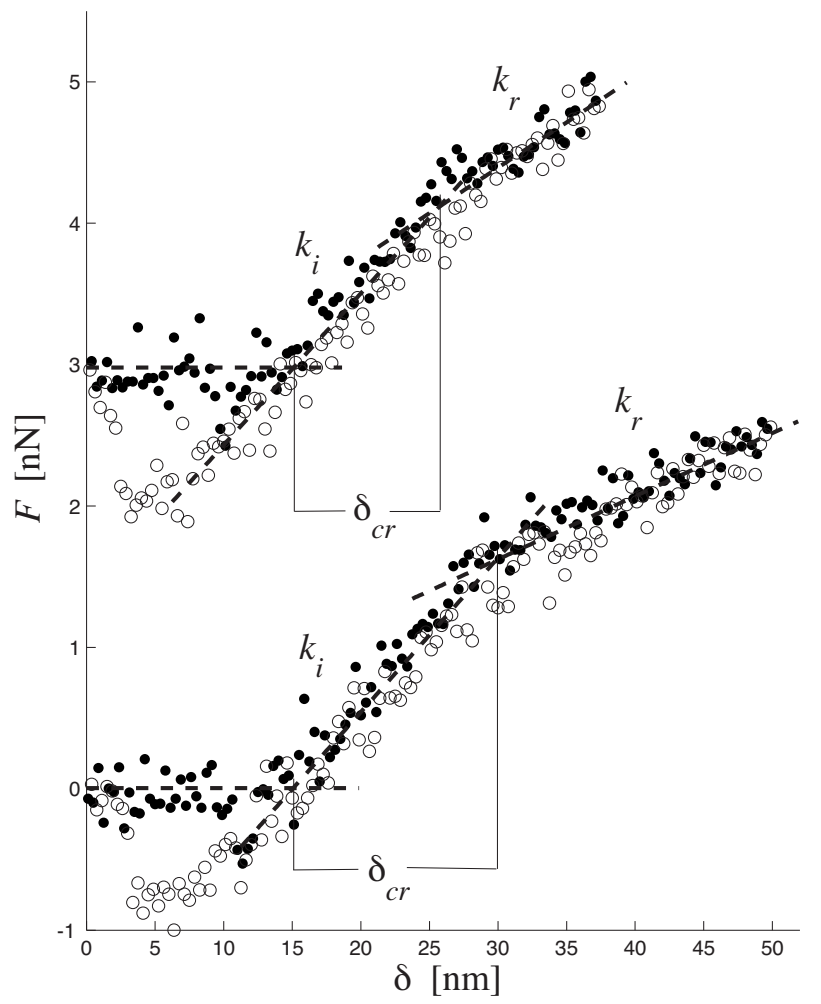

FIG. 2. Force vs displacement curves for two individual nanotubes in the forward direction ( ) and backward direction ( $\bigcirc)$. The slopes before, $k_{i}$, and during rippling, $k_{r}$, are indicated as well as the critical displacement $\delta_{c r}$. The dimensions of the measured nanotubes were $r=3.75 \mathrm{~nm}, l=99.6 \mathrm{~nm}$ (top curve), and $r=3.55 \mathrm{~nm}, l=65.6 \mathrm{~nm}$ (bottom curve). for thicker tubes. In measurements on supported tubes, Wong et al. ${ }^{11}$ have seen a similar ratio, about 0.4 as measured in their Fig. 4, indicating that they may well have seen rippling rather than the suggested buckling. In resonant measurements ${ }^{8}$ a mix of $k_{r}$ and $k_{i}$ might be involved for large diameter tubes, thus yielding a lower bending stiffness and a misinterpretation of $E$. The ratio $k_{r} / k_{i}$ that we have observed here is however too low to fully explain the large spread in $E$ that has been reported. ${ }^{8}$

The critical displacements obtained from the $F-\delta$ curves were used to calculate $\varepsilon_{c r}$, which for a cantilevered beam of circular cross section with radius $r$, and length $l$, is given by:

$$
\varepsilon_{c r}=\frac{3 \delta_{c r} r}{l^{2}}
$$

where both $r$ and $l$ were obtained from SEM images. ${ }^{14}$ Early theoretical work ${ }^{6}$ has suggested that $\varepsilon_{c r}=0.006$, i.e., that the critical strain is a material constant. Later simulations 3,7 found that $\varepsilon_{c r}$ should depend on $r$ as $\varepsilon_{c r}=l_{c r} / r$, where the constant $l_{c r}$ (coined the critical length ${ }^{3}$ ) was different in the two articles. Arias and Arroyo ${ }^{3}$ found $l_{c r}=0.1$ and Nikiforov et al. ${ }^{7}$ found $l_{c r}=0.05$. Our obtained values for $\varepsilon_{c r}$ are plotted in Fig. 3(a) versus the radius. For comparison the three theoretical predictions are plotted as solid, ${ }^{6}$ dashed, ${ }^{3}$ and dotted-dashed $^{7}$ lines, respectively.

From the $F$ - $\delta$ curves the initial spring constant, $k_{i}$, can be used to evaluate the inherent Young's modulus. By using beam theory for a cantilevered hollow cylinder we have:

$$
E=\frac{4 k_{i} l^{3}}{3 \pi r_{o}^{4}} \times \frac{r_{o}^{4}}{r_{o}^{4}-r_{i}^{4}},
$$

where $r_{i}$ and $r_{o}$ are the inner and outer radii, respectively. By using TEM we have found that most tubes had ratios $r_{i} / r_{o}$ below 0.5 . The second term in Eq. (2) is thereby less than 1.07 and can be neglected. Resulting values of $E$ are plotted in Fig. 3(b) versus $r$. The obtained values are well below the highest observed values of $E=1 \mathrm{TPa}$ for arc discharge grown tubes. ${ }^{17}$ Lower $E$ values are commonly observed though for CCVD grown CNTs (Ref. 17) where the growth process can leave defects in the nanotubes, thus lowering $E$.

As can be seen in Fig. 3(a) the values for $\varepsilon_{c r}$ have a large spread for similar radii. With an uncertainty of $20 \%$ in $\delta_{c r}$, $10 \%$ in $r$, and $10 \%$ in $l$, the resulting spread in $\varepsilon_{c r}$ would be about a factor of 3 . The measured values are scattered at least 

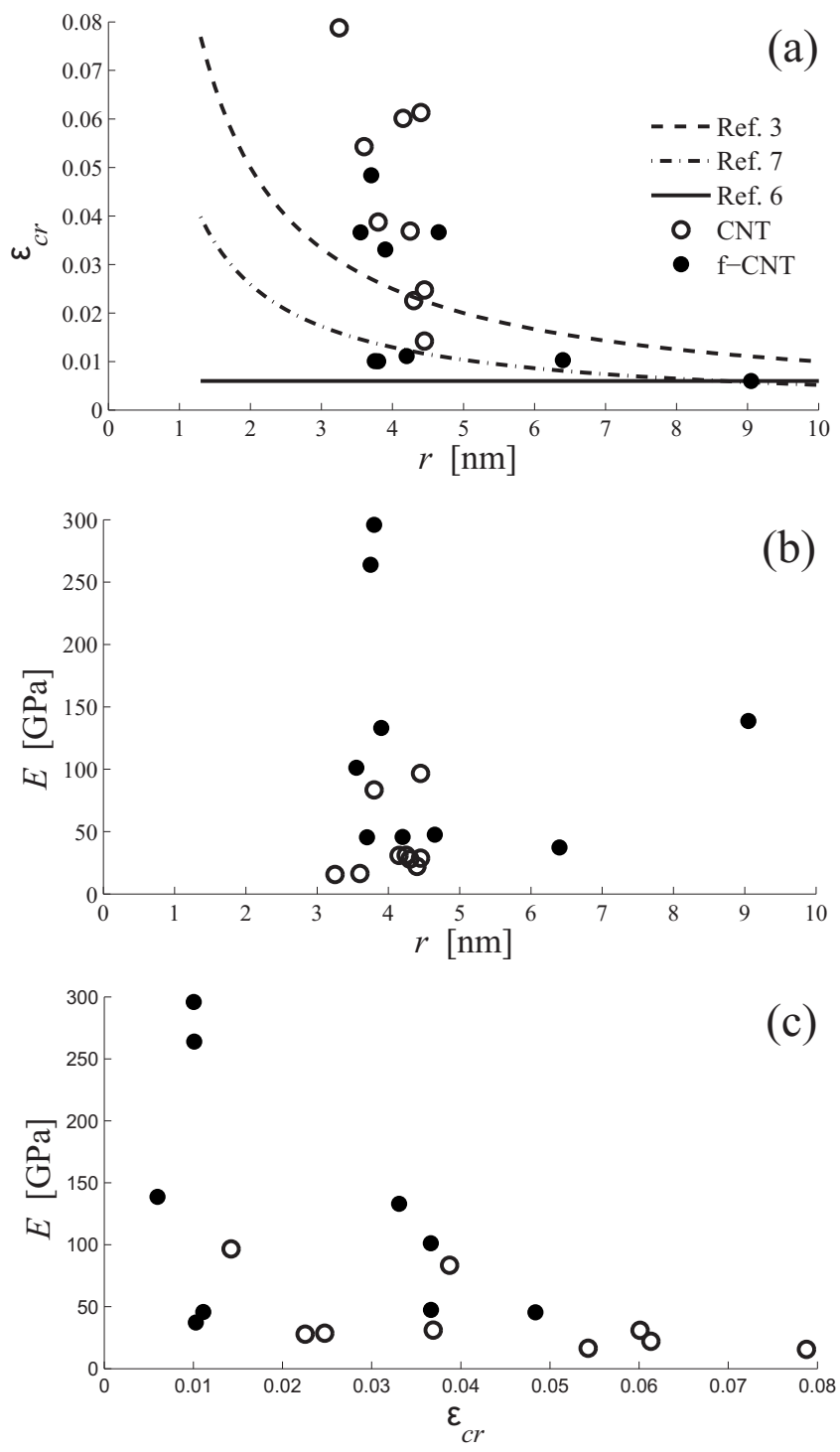

FIG. 3. (a) Measured critical strain for rippling, $\varepsilon_{c r}$, vs the radius of the unfunctionalized $(\bigcirc)$ and the functionalized CNTs $(-)$. (b) Young's modulus vs the radius of the CNTs (obtained from $k_{i}$ ). (c) Young's modulus vs the critical strain.

by a factor of 6 , which cannot be explained solely with the uncertainties of our measurements, but rather due to varying properties of the CNTs.

The theoretical predictions of $\varepsilon_{c r}$ plotted in Fig. 3(a) all assume perfect defect-free CNTs with weak van der Waals forces between layers. The influence of interwall covalent bridges on the critical strain has been modeled ${ }^{18}$ and the results showed that $\varepsilon_{c r}$ increases with higher density of in- terwall covalent bridges. Such covalent bridges stem from defects in the nanotubes and as mentioned CCVD grown CNTs have an inherent large defect density. A spread in $E$ by an order of magnitude for approximately the same $r$ has been reported, ${ }^{17}$ indicating varying defect densities for CCVD grown nanotubes. We have found a similar spread, as seen in Fig. 3(b), and by plotting $E$ versus $\varepsilon_{c r}$ in Fig. 3(c) we have found indications that the CNTs which are more resistant to rippling (large $\varepsilon_{c r}$ ) are also the ones that have smaller $E$ values.

In conclusion, we have been able to experimentally detect the onset of the rippling mode in free standing CNTs. The change in spring constant associated with rippling was found to be independent of the tubes radius and we have found a mean value of 0.56 for the ratio, $k_{r} / k_{i}$. We have also found indications that $\varepsilon_{c r}$ increases with higher defect densities, and it is accompanied by a drop in Young's modulus. So while intentional introduction of defects might be a route to avoid rippling in devices, there would also be a drop in Young's modulus, which is larger than the reduction caused by rippling alone.

Financial support from the Swedish Research Council (project number 2010-4324) is gratefully acknowledged.

${ }^{1}$ J. M. Kinaret, T. Nord, and S. Viefers, Appl. Phys. Lett. 82, 1287 (2003).

${ }^{2}$ A. B. Kaul, E. W. Wong, L. Epp, and B. D. Hunt, Nano Lett. 6, 942 (2006).

${ }^{3}$ I. Arias and M. Arroyo, Phys. Rev. Lett. 100, 085503 (2008).

${ }^{4}$ M. Arroyo and T. Belytschko, Phys. Rev. Lett. 91, 215505 (2003).

${ }^{5}$ M. Arroyo and I. Arias, J. Mech. Phys. Solids 56, 1224 (2008).

${ }^{6}$ J. Liu, Q. Zheng, and Q. Jiang, Phys. Rev. B 67, 075414 (2003).

${ }^{7}$ I. Nikiforov, D.-B. Zhang, R. D. James, and T. Dumitrică, Appl. Phys. Lett. 96, 123107 (2010).

${ }^{8}$ P. Poncharal, Z. L. Wang, D. Ugarte, and W. A. de Heer, Science 283, 1513 (1999)

${ }^{9}$ S. Iijima, C. Brabec, A. Maiti, and J. Bernholc, J. Chem. Phys. 104, 2089 (1996)

${ }^{10}$ D. Qian, W. K. Liu, S. Subramoney, and R. S. Ruoff, J. Nanosci. Nanotechnol. 3, 185 (2003).

${ }^{11}$ E. W. Wong, P. E. Sheehan, and C. M. Lieber, Science 277, 1971 (1997)

${ }^{12}$ A. Nafari, D. Karlen, C. Rusu, K. Svensson, H. Olin, and P. Enoksson, J. Microelectromech. Syst. 17, 328 (2008).

${ }^{13}$ Identical to their controller for in situ transmission electron microscopy instruments.

${ }^{14}$ See supplementary material at http://dx.doi.org/10.1063/1.3587613 for more information on how the AFM has been calibrated and how the diameters and lengths of the CNTs have been determined.

${ }^{15}$ More information about the CNTs used in this study can be found at www.nanocyl.com.

${ }^{16}$ K. Svensson, Y. Jompol, H. Olin, and E. Olsson, Rev. Sci. Instrum. 74, 4945 (2003).

${ }^{17}$ K. Lee, B. Lukić, A. Magrez, J. W. Seo, G. A. D. Briggs, A. J. Kulik, and L. Forró, Nano Lett. 7, 1598 (2007).

${ }^{18}$ X. Huang and S. Zhang, Appl. Phys. Lett. 96, 203106 (2010). 